\title{
Impacto del uso de los recursos tecnológicos en el rendimiento académico
}

\section{Impact of the use of technological resources on academic performance}

Cynthia Martínez-Garrido

Universidad Autónoma de Madrid, España (cynthia.martinez@uam.es)

Recibido el 8 de junio de 2018; revisado el 7 de octubre de 2018; aceptado el 22 de octubre de 2018; publicado el 1 de diciembre de 2018

\section{RESUMEN:}

El uso de los recursos educativos en las lecciones ha sido destacado por la investigación sobre enseñanza eficaz como uno de los factores clave que hacen que la docencia sea de calidad. La presente investigación busca describir la utilización que los estudiantes de Educación Secundaria dan a los recursos tecnológicos dentro y fuera de la escuela y determinar la relación entre la utilización de los recursos tecnológicos dentro de la escuela y el rendimiento académico en matemáticas y lectura. Para dar respuesta a ambos objetivos se lleva a cabo una explotación secundaria de la base de datos PISA 2015 con los datos de 6736 estudiantes de 201 escuelas de Educación Secundaria Obligatoria en España. Como método de análisis de datos se utilizan los análisis multinivel. Los resultados señalan que los recursos tecnológicos están presentes en el día a día de los estudiantes tanto fuera como dentro de las escuelas y que la utilización del correo electrónico, la conexión a Internet y el uso del ordenador generan mejoras en el desarrollo académico de los estudiantes, tanto en matemáticas y lectura. El compromiso con una enseñanza de calidad está ligado al uso de los recursos tecnológicos; por ello, es fundamental que su utilización ajustada al contexto y estudiantes en todas las escuelas.
PALABRAS
CLAVE:
RECURSOS
EDUCACIONALES; TECNOLOGÍAS DE LA INFORMACIÓN; RENDIMIENTO ESCOLAR; MATEMÁTICAS; LECTURA.

\begin{abstract}
The use of educational resources in the classrooms has been highlighted by the research on Effective Teaching as one of the key factors that enhance the quality of teaching. The aims of this research is to describe the use that Secondary Education students give to technological resources inside and outside the school and determine the relationship between the use of technological resources within the school and the academic performance of students in mathematics and reading. To do so, it's been conducted a secondary exploitation of the PISA 2015 database, which include the data of 6736 students from 201 compulsory secondary education schools in Spain. Multilevel analyzes are used as a method of data analysis. The results indicate that technological resources are present in the students' day-to-day activities both outside and inside the schools, and that the use of email, the connection to the Internet and the use of the computer generate improvements in the academic development of students in mathematics and reading. The commitment to quality education is linked to the use of technological resources; therefore, it is essential that the adjusted use of technological resources be strengthened in all schools.
\end{abstract}

$\begin{array}{lrr}\text { KEYWORDS: } & \text { OPEN } & \text { EDUCATIONAL } \\ \text { RESOURCES; } & \text { SCIENTIFIC } & \text { INFORMATION; } \\ \text { ACADEMIC } & & \text { ACHIEVEMENT; }\end{array}$




\section{INTRODUCCIÓN}

Una de las líneas de investigación educativa más importante, por el impacto que sus resultados han generado sobre la toma de decisiones de los sistemas educativos en todo el mundo, las escuelas y las aulas, es la conocida como investigación sobre investigación sobre eficacia educativa (educacional effectiveness research) (Murillo, Martínez-Garrido y Hernández-Castilla, 2011; Murillo, 2007; Scheerens, 2013). Entre otras, esta línea de investigación ha aportado interesantes conocimientos acerca de los elementos de la escuela $\mathrm{y}$ de las aulas, que contribuyen al desarrollo holístico de los estudiantes; en definitiva, a que aprendan más y mejor.

Concretamente, la investigación educativa que estudia los elementos que favorecen el aprendizaje de todos los estudiantes, que definen la calidad de la labor de los docentes, de su enseñanza y del aula en su conjunto, es la conocida como investigación sobre enseñanza eficaz (teaching effectiveness research). Esta línea de investigación ha identificado el aula como el lugar por excelencia donde se producen los aprendizajes. Sus postulados parten de la consideración de que todos pueden aprender, solo es necesario tener los elementos para que el aprendizaje se dé lo más eficazmente (Creemers, 1994; Martínez-Garrido y Murillo, 2016; Reynolds et al., 2014).

\section{REVISIÓN DE LA LITERATURA}

El uso de los recursos educativos en las aulas ha sido destacado por la investigación sobre enseñanza eficaz como uno de los factores clave que hacen que la docencia sea de calidad. La investigación ha demostrado que los profesores que consiguen que sus estudiantes aprendan más y mejor apoyan su docencia con la utilización de recursos didácticos variados (Hammond, 2014). La clave, por tanto, está en identificar cuáles son esos recursos y qué uso se da a los mismos.

Quizá, uno de los trabajos más representativos en abordar el impacto de los recursos educativos es el macroestudio realizado por Cervini (2009) con datos de casi 300.000 estudiantes de 4.208 escuelas argentinas. En este trabajo el autor investiga cuál es la influencia de los recursos utilizados por docentes durante las lecciones sobre el rendimiento en matemáticas. Concretamente, se analiza la disponibilidad y condiciones de doce tipologías de recursos pedagógicos: libros de los maestros, las revistas de actualización pedagógica, textos, libros y guías para los estudiantes, vídeo didáctico, proyector, retroproyector, cinta grabadora de vídeo y material de laboratorio. Los resultados muestran que el impacto de los recursos educativos sobre el rendimiento en matemáticas explica el 17\% de la variación del logro alcanzado por los estudiantes de los diferentes municipios que participaron en el estudio.

La llegada de las tecnologías de la información y la comunicación (TIC) a las escuelas obliga a la construcción de un nuevo contexto cultural y la adaptación global de la propia escuela, pero sobre todo de los docentes. Como señala Marqués (2013), las escuelas pueden optar por realizar pequeños ajustes en el currículum para que se utilicen los recursos tecnológicos como fuente de información (escenario tecnócrata), pueden además introducir este tipo de recursos en la metodología docente (escenario reformista) o pueden llegar a reestructurar los elementos de la escuela de manera global haciendo que la actividad de toda la escuela cambie (escenario holístico). Tres planteamientos de mejora de la escuela que profundizan en las posibilidades que los recursos tecnológicos abren a la docencia en las aulas.

La formación y la preparación de los docentes son aspectos que se convierten en prioritarios para hacer un buen uso de las innovaciones, más si cabe cuando se trata de la inclusión de los recursos tecnológicos. Estudios como los desarrollados por Agodini, Dynarski, Honey y Levin (2003), Balanskat, Blamire y Kefala (2006), Pérez y Sola (2006), o Area (2007), entre otros, señalan que para obtener el máximo beneficio de la implantación de las tecnologías es necesario i) formación a los docentes en torno al uso y manejo de las herramientas, ii) formación didáctica que facilite la introducción del recurso de manera normalizada en el aula y les permita generar conocimientos abiertos al análisis, la reflexión y al cambio, y iii) orientación docente que les sitúe como organizadores, orientadores y supervisores de actividades de aprendizaje de los estudiantes gracias a los recursos utilizados.

Los beneficios de la integración de los recursos tecnológicos en la docencia en la Educación Secundaria están bien documentados por la literatura (Fernández-Miravete, 2018; Garrison y Bromley, 2004; Huertas Montes y Pantoja Vallejo, 2016; Reedy, 2008; Pantoja y Villanueva, 2015). Sosa, Peligros y Dionisio Díaz (2010) demuestran que la integración de los recursos tecnológicos en la didáctica de las materias favorece la interrelación entre el estudiante, la información y su uso 
inteligente. Según Lizasoain y Joaristi (2012), los recursos tecnológicos desarrollan habilidades cognitivas, de análisis, síntesis, aplicación y evaluación de la información, además de favorecer la conexión de ideas y la creación de nuevos conocimientos. El estudio de Genlott y Grönlund (2016) apunta que la interactividad que conlleva el uso de los recursos tecnológicos en las tareas favorece la adquisición de un aprendizaje más significativo y profundo, además de permitir la puesta en marcha de actividades en espacios virtuales.

Otros trabajos sugieren que, aunque la gran mayoría de los estudiantes actualmente utilizan los recursos tecnológicos al interior de la escuela, los patrones de interacción sostenida y variada con la tecnología continúa difiriendo dentro y fuera de las escuelas (Selwyl, Potter y Cranmer, 2008). Las dificultades en la integración de las tecnologías por parte de los docentes de la escuela, o del acceso a ellas, son los elementos que limitan los efectos positivos de su integración en la docencia.

Efectivamente, la investigación es contundente en afirmar que las bondades de los recursos tecnológicos no se producen por el simple hecho de integrarlos de manera sistemática en la docencia (Aldama y Pozo, 2016). Aunque los recursos tecnológicos son cada vez más frecuentes en las escuelas, sus efectos positivos se merman si se utilizan como meros instrumentos de apoyo y refuerzo de los conocimientos previos (Valverde-Berrocoso y Sosa-Díaz, 2010). Así, la integración en el currículum de las tecnologías y el uso que se dé de ellas en el proceso de enseñanza y aprendizaje determina los efectos positivos de la innovación. En un estudio previo Paiva (2005), con los datos de 8.140 estudiantes de Educación Secundaria en Portugal, identificó que el $43 \%$ de los estudiantes utilizaba el ordenador exclusivamente para juegos no relacionados con el currículo. Y que tan solo el $30 \%$ y el $18 \%$ de los estudiantes se conectaba a Internet para jugar a juegos educativos y enviar correos electrónicos, respectivamente.

En España, los recursos tecnológicos tienen una presencia importante en las escuelas. De acuerdo con el reciente estudio de Sánchez-Antolín y Blanco-García (2016), al menos $85 \%$ de las escuelas tienen acceso Internet en todas las aulas, un $80 \%$ tienen al menos un ordenador para el profesor y más del $50 \%$ de las aulas tienen un ordenador para cada estudiante. Sin embargo, los recursos más novedosos, como son las tabletas, son minoritarios; tan solo el $0,8 \%$ de las aulas dispone de ellos. También está generalizada la presencia de los recursos tecnológicos en los hogares. Según Gairín Sallán y Mercader (2018) el 95\% y 93\% de las familias españolas tienen Internet en casa y ordenador, respectivamente.

Entre los estudios más recientes realizados sobre el uso de los recursos tecnológicos en España destacan aquellos que abordan el análisis de materiales digitales educativos, la utilización de los portales web educativos, la formación digital del profesorado o el impacto de las políticas educativas TIC en las escuelas (Baztan, 2014; Bonilla, Nemiña y Suelves, 2017; Sánchez-Antolín y Blanco-García, 2016). Por ejemplo, el estudio realizado por Sánchez-Antolín y Blanco-García (2016) analiza el impacto que generan sobre la organización y la docencia en las escuelas de las políticas 1:1 instauradas por la Comunidad de Madrid, por las que se regula la inclusión de ordenadores de mesa en las aulas de $1^{\circ}$ y $2^{\circ}$ de la ESO. De acuerdo con los autores, los docentes indican una fuerte desconexión en torno a la forma de agrupar al alumnado que exige este tipo de innovaciones y las medidas de atención a la diversidad en las aulas.

Por su parte, los resultados obtenidos por Pantoja Villanueva (2015) y Huertas Montes y Pantoja Vallejo (2016) son especialmente relevantes para los objetivos de la presente investigación. De un lado, el estudio realizado por Pantoja y Villanueva (2015) comprueba que los programas educativos basados en la utilización de recursos tecnológicos generan efectos positivos sobre los estudiantes más allá de sobre su motivación, concretamente, en el conocimiento de la propia cultura y la de sus compañeros, y sobre el desarrollo de valores democráticos y ciudadanos. De otro, Huertas Montes y Pantoja Vallejo (2016) analizan, a través de un estudio experimental, la influencia de los recursos tecnológicos sobre el rendimiento académico y la motivación de los estudiantes, además de analizar el uso que los docentes realizan de las tecnologías en las aulas. Según los autores, los estudiantes que utilizan los recursos tecnológicos en las aulas no solo obtienen mejores calificaciones sino también están más motivados.

A la luz de la revisión realizada, esta investigación analiza la relación entre la utilización de los recursos tecnológicos y el rendimiento académico de los estudiantes en España utilizando los datos PISA 2015. Concretamente, la investigación persigue dos objetivos específicos: 
1. Describir la utilización que los estudiantes de Educación Secundaria dan a los recursos tecnológicos dentro y fuera de la escuela.

2. Determinar la relación entre la utilización de los recursos tecnológicos dentro de la escuela y el rendimiento académico de los estudiantes en matemáticas y lectura.

\section{MÉTODO}

Para dar respuesta a estos objetivos se realiza una explotación secundaria de la base de datos del Programa Internacional de Evaluación de los Alumnos (PISA) de la OCDE, correspondiente a 2015, último disponible. El programa PISA evalúa, de forma trienal desde el año 2000, los resultados de los sistemas educativos de diversos países en tres áreas fundamentales: Matemáticas, Lengua y Ciencias; y obtiene información de factores asociados al aprendizaje que permite contextualizar $\mathrm{y}$, hasta cierto punto, explicar los resultados obtenidos. Para la recogida de datos, PISA utiliza, aparte de las pruebas de rendimiento, cuestionarios de contexto dirigidos a los directores de los centros, las familias y los estudiantes de educación secundaria.

Esta investigación se trabaja con los datos de matemáticas y lectura analizándolos con el enfoque de modelos multinivel con dos niveles de análisis: estudiante y escuela. Las variables utilizadas son de tres tipos: de producto, de ajuste y explicativas o independientes.

- Variables de producto:

- Rendimiento en matemáticas: mide la alfabetización matemática para formular, emplear e interpretar las matemáticas en una variedad de contextos para describir, predecir y explicar fenómenos, reconociendo el papel que juegan las matemáticas en el mundo. Media 500, desviación típica 100.

- Rendimiento en lectura: mide la capacidad de entender, utilizar y reflexionar sobre textos escritos con el fin de lograr los objetivos, desarrollar el conocimiento y potencial, y participar en la sociedad. Media 500, desviación típica 100.

- Variables de ajuste:

- Nivel socioeconómico y cultural de la familia, variable tipificada. Este índice se compone de índices parciales relacionados con el contexto socioeconómico y cultural del estudiante (índice del mayor nivel educativo de los padres, índice del mayor nivel laboral de los padres, e índice del patrimonio cultural familiar). El índice NSEC, para el total de los países que participan en PISA, tiene un valor medio de cero y una desviación típica de uno. De manera que una puntuación negativa indica que las familias de los alumnos de un país tienen un nivel socioeconómico y cultural inferior a la media de los países participantes en PISA (OCDE, 2016).

- Nivel socioeconómico y cultural de la escuela, variable tipificada.

- Género del estudiante, variable dummy (0, varón).

- Inmigrante, variable dummy ( 0 , nativo)

- Asistencia a preescolar, número de años que asistió a algún centro educativo antes de comenzar la educación obligatoria.

Variables explicativas o independientes:

- Chat para hablar con los compañeros, frecuencia con la que el estudiante utiliza los recursos tecnológicos para chatear con sus compañeros de clase.

- Correo electrónico para compartir trabajos con estudiantes y/o profesores, frecuencia con la que el estudiante utiliza los recursos tecnológicos para enviar correos electrónicos sobre trabajos escolares

- Internet para hacer los trabajos escolares, frecuencia con la que el estudiante utiliza los recursos tecnológicos para conectarse a Internet y consultar información para sus trabajos escolares.

- Internet para descargar información, frecuencia con la que el estudiante utiliza los recursos tecnológicos para descargarse documentación.

- Plataforma web del colegio para subir trabajos escolares, frecuencia con la que el estudiante utiliza los recursos tecnológicos para cargar sus trabajos en la web de la escuela

- Simulaciones educativas, frecuencia con la que el estudiante utiliza los recursos tecnológicos para jugar a simulaciones relativas al currículo.

- Ordenador para hacer ejercicios del currículo, frecuencia con la que el estudiante utiliza los recursos tecnológicos para realizar ejercicios didácticos propuestos por el docente.

- Ordenador para hacer los deberes, frecuencia con la que el estudiante utiliza los 
recursos tecnológicos para hacer los deberes extraescolares.

- Ordenador para trabajar en grupo, frecuencia con la que el estudiante utiliza los recursos tecnológicos para trabajar en grupo con los compañeros.

Para la selección de la muestra, PISA utiliza un muestreo estratificado por conglomerados en dos etapas, con muestras en la primera etapa proporcionales al tamaño. Las unidades primarias de muestreo son las escuelas y las secundarias los alumnos de cada escuela, que se seleccionaron 35 de forma aleatoria a partir de listas de sujetos elegibles en cada escuela. Las variables de estratificación explícitas utilizadas para el caso español son la titularidad del centro, comunidad autónoma, tamaño de la escuela y modalidad de enseñanza en el País Vasco; las implícitas, los códigos postales y en Cataluña el tamaño de la localidad. La selección efectiva de la muestra se lleva a cabo mediante un procedimiento sistemático con un intervalo de muestreo. La distribución de estudiantes y escuelas de la muestra se encuentra en la tabla 1.

Tabla 1. Muestra del estudio. Número de estudiantes, de centros y \% de inmigrantes

\begin{tabular}{lccr}
\hline & ESTUD. & CENTROS & \% INMIG \\
\hline Andalucía & 1.813 & 54 & 3,23 \\
Aragón & 1.798 & 53 & 12,45 \\
Ppdo. Asturias & 1.790 & 54 & 5,79 \\
Illes Balears & 1.797 & 54 & 14,24 \\
Canarias & 1.842 & 54 & 9,60 \\
Cantabria & 1.924 & 56 & 9,00 \\
Castilla y León & 1.858 & 57 & 6,60 \\
Cast.-La Mancha & 1.889 & 55 & 7,63 \\
Cataluña & 1.769 & 52 & 14,81 \\
Extremadura & 1.809 & 53 & 1,74 \\
Galicia & 1.865 & 59 & 5,07 \\
La Rioja & 1.461 & 47 & 15,70 \\
C. de Madrid & 1.808 & 51 & 14,84 \\
Reg. de Murcia & 1.796 & 53 & 12,77 \\
C. F. Navarra & 1.874 & 52 & 13,10 \\
País Vasco & 3.612 & 119 & 7,75 \\
C. Valenciana & 1.625 & 53 & 12,28 \\
\hline Total España & 6.736 & 201 & 9,07 \\
\hline
\end{tabular}

Fuente: Elaboración propia

Se utilizaron distintas estrategias de análisis datos. Así, para abordar el uso que los jóvenes dan a los recursos didácticos se realizaron análisis descriptivos. Y, por otro lado, se han utilizado estadísticos más complejos para determinar el impacto que genera la utilización de los recursos tecnológicos en el desarrollo cognitivo de los estudiantes; en este caso, se usaron los modelos multinivel de dos niveles de análisis. La técnica de análisis de datos multinivel está recomendada especialmente para este tipo de estudios con datos anidados (Martínez-Garrido y Murillo, 2016).

El procedimiento para cada una de las variables producto fue:

- Estimar el modelo nulo (modelo I), solo con la variable producto.

- Calcular el modelo con las variables de ajuste (modelo II).
- Incluir en el modelo ajustado las variables referidas a la utilización de los recursos tecnológicos (modelo III).

- Calculo del modelo final con las variables de ajuste y con tan solo aquellas variables que han resultado significativas durante el proceso de modelado descrito anteriormente (modelo IV).

De esta forma se estimaron dos modelos multinivel (uno para cada variable de producto) análogos al siguiente:

$$
\begin{aligned}
& Y_{i j}=\beta_{0 i j}+\beta_{1 j} N S E \_ \text {esc }{ }_{j}+\beta_{2 j} N S E C \_f a m_{i j}+ \\
& \beta_{3 j} \text { Genero }_{i j}+\beta_{4 j} \text { Inmigrante }_{i j}+\beta_{4 j} \text { Preescolar }_{i j}+ \\
& +\beta_{5 \mathrm{j}} \mathrm{F}_{-}{ }_{C h a t} \mathrm{t}_{\mathrm{j} j}+\beta_{6 \mathrm{j}} \mathrm{F} \_ \text {Email }{ }_{\mathrm{ij}}+\beta_{7 \mathrm{j}} \mathrm{F}{ }_{-} \text {Consultar_i } \\
& \text { nternet }_{i j}+\beta_{8 j} F_{-}{ }_{\text {Descargas }}{ }_{i j}+ \\
& +\beta_{9 \mathrm{j}} \mathrm{F} \_ \text {Subir_trabajos }{ }_{\mathrm{ij}}+\beta_{10 \mathrm{j}} \mathrm{F}_{-} \mathrm{Juegos}_{\mathrm{ij}}+\beta_{1 \mathrm{j}} \mathrm{F}_{-} \\
& \text {Ejercicios }_{\mathrm{ijj}}+\beta_{12 \mathrm{j}} \mathrm{F} \_ \text {Deberes }_{\mathrm{ij}}+ \\
& +\beta_{13 j} F_{-} \text {Trabajo_grupal }{ }_{i j}+e_{i j}
\end{aligned}
$$


$\beta_{0 \mathrm{ij}}=\beta_{0}+\mathrm{u}_{0 \mathrm{j}}+\mathrm{e}_{0 \mathrm{ij}}$

$\beta_{1 \mathrm{j}}=\beta_{1}+\mathrm{u}_{1 \mathrm{j}} \ldots \beta_{12 \mathrm{j}}=\beta_{12}+\mathrm{u}_{12 \mathrm{j}}$

donde:

$Y_{i j=}$ rendimiento alcanzado en Matemáticas o Lectura.

$\beta_{0 i j}=$ Es el promedio de $Y_{i j}$ de la escuela $\mathrm{j}$ ésima para el estudiante $\mathrm{i}$

$\beta_{0}=$ El promedio de $Y_{i j}$ para la población

$\mu_{0 j}=$ Es el efecto aleatorio asociado a la escuela jésima, con distribución normal, media 0 y varianza $\Omega_{\mu}$

$e_{0 i j}=$ Es el efecto aleatorio asociado al estudiante $\mathrm{i}$, con distribución normal, media 0 y varianza $\Omega_{e}$

NSE_esc , nivel socioeconómico del barrio donde se encuentra la escuela.

NSE_fam ${ }_{\mathrm{ij}}$, nivel socioeconómico de la familia del estudiante.

Genero $_{i j}$, género del estudiante

Inmigrante $_{\mathrm{ij}}$, si el estudiante es inmigrante o no.

Preescolar $_{\mathrm{ij}}$, si el estudiante a acudido a Educación preescolar

F_Chat $t_{i j}$, frecuencia con la que el estudiante utiliza los recursos tecnológicos para chatear F_Email $1_{\mathrm{ij}}$, frecuencia con la que el estudiante utiliza los recursos tecnológicos para mandar correos electrónicos

F_Consultar_internet ${ }_{i j}$, frecuencia con la que el estudiante utiliza los recursos tecnológicos para

F_Descargas $_{\mathrm{ij}}$, frecuencia con la que el estudiante utiliza los recursos tecnológicos para descargar documentación

F_Subir_Trabajos $\mathrm{ij}_{\mathrm{ij}}$, frecuencia con la que el estudiante utiliza los recursos tecnológicos para cargar sus trabajos en la web de la escuela

F_Jugar ${ }_{i j}$, frecuencia con la que el estudiante utiliza los recursos tecnológicos para jugar a juegos educativos
F_Ejercicios $\mathrm{ij}_{\mathrm{ij}}$ frecuencia con la que el estudiante utiliza los recursos tecnológicos para hacer ejercicios.

F_Deberes ${ }_{\mathrm{ij}}$, frecuencia con la que el estudiante utiliza los recursos tecnológicos para hacer los deberes extraescolares.

F_Trabajo_grupal $\mathrm{i}_{\mathrm{ij}}$, frecuencia con la que el estudiante utiliza los recursos tecnológicos para para trabajar en grupo con los compañeros.

\section{RESULTADOS}

De acuerdo con los objetivos del estudio, se han agrupado los resultados obtenidos bajo dos grandes epígrafes. En el primero de ellos se aborda el estudio acerca de cómo los recursos tecnológicos están siendo utilizados, tanto dentro como fuera de la escuela. Y, a continuación, se presenta la estimación del efecto que el uso de los recursos tecnológicos en la escuela genera sobre el rendimiento académico de los estudiantes.

\subsection{Utilización de los recursos tecnológicos}

Los estudiantes utilizan los recursos tecnológicos fuera de la escuela abordando cuestiones que guardan relación con el currículum académico (tabla 2). Concretamente, las dos utilidades más frecuentes son la utilización de las redes sociales para comunicarse con otros compañeros de clase sobre temas escolares y conectarse a Internet para hacer los trabajos escolares. Los datos indican que el $66 \%$ y el $64 \%$ de los estudiantes afirma conectarse a Internet para consultar dudas y preparar y estudiar las lecciones, respectivamente. Aunque, el 50\% de los estudiantes dice realizarla al menos varias veces semanalmente. 
Tabla 2. Frecuencia de utilización de los recursos tecnológicos fuera de la escuela, en porcentaje

\begin{tabular}{lcccc}
\hline & CASI NUNCA & MENSUALMENTE & SEMANALMENTE & A DIARIO \\
\hline $\begin{array}{l}\text { Conectarme a internet para hacer trabajos } \\
\quad \text { escolares }\end{array}$ & 7,17 & 29,34 & 37,43 & 26,06 \\
$\begin{array}{l}\text { Conectarme a Internet para consultar preparar los } \\
\quad \text { temas y resolver dudas }\end{array}$ & 23,27 & 27,34 & 28,90 & 20,49 \\
$\begin{array}{l}\text { Mandar correos electrónicos a compañeros sobre } \\
\quad \text { trabajos escolares }\end{array}$ & 40,52 & 24,15 & 19,22 & 16,11 \\
$\begin{array}{l}\text { Mandar correos electrónicos al profesor o enviar } \\
\quad \text { deberes }\end{array}$ & 39,31 & 33,15 & 16,77 & 10,90 \\
$\quad \begin{array}{l}\text { Uso de redes sociales para hablar con otros } \\
\quad \text { compañeros sobre trabajos escolares }\end{array}$ & 21,64 & 12,68 & 20,21 & 45,46 \\
$\quad \begin{array}{l}\text { Uso de redes sociales para hablar con los } \\
\quad \text { profesores }\end{array}$ & 73,84 & 9,29 & 6,46 & 10,42 \\
$\begin{array}{l}\text { Descargar materiales de la web de la escuela } \\
\text { Visitar la web de la escuela para consultar avisos }\end{array}$ & 48,48 & 23,72 & 16,04 & 11,76 \\
$\quad$ y anuncios & 52,46 & 20,55 & 14,77 & 12,22 \\
$\quad \begin{array}{l}\text { Usar en el ordenador para hacer los deberes } \\
\quad \text { escolares }\end{array}$ & 37,48 & 26,23 & 20,93 & 15,36 \\
$\quad \begin{array}{l}\text { Usar la tableta/télefono móvil para hacer los } \\
\quad \text { deberes }\end{array}$ & 61,55 & 15,56 & 11,24 & 10,47 \\
Descargar aplicaciones de enseñanza & 69,67 & 15,50 & 7,47 & 7,35 \\
\hline
\end{tabular}

Por su parte, mandar correos electrónicos entre los compañeros de clase es la siguiente utilidad más frecuente (el $35 \%$ lo hace al menos varias veces a la semana), aunque destaca que el $40 \%$ de los estudiantes afirmen no hacerlo nunca. Es relevante la baja frecuencia del uso de la plataforma web de la escuela entre los estudiantes, el 52\% afirma no consultarla casi nunca y el $48 \%$ raras veces se descarga materiales de ella. Los usos menos frecuentes entre los estudiantes son la comunicación con los docentes a través de las redes sociales (solo un $17 \%$ afirma hacerlo con mucha frecuencia) y descargarse aplicaciones informáticas educativas (el 15\%, de los cuales solo el $3 \%$ indica hacerlo de manera muy habitual) (tabla 2).

$\mathrm{Al}$ interior de las escuelas, consultar Internet para realizar los trabajos encargados por el docente continúa siendo la actividad más frecuente según los estudiantes (44\%). Y, de nuevo, utilizar aplicaciones educativas y utilizar la plataforma web de la escuela son los usos menos frecuentes, el $75 \%$ y el $72 \%$, respectivamente de los estudiantes confirma no hacerlo casi nunca. Los recursos tecnológicos no solo son utilizados para el desarrollo del estudiante de manera individual, también para el trabajo en grupo. Aunque el $44 \%$ de los estudiantes afirma no utilizarlos casi nunca en grupo, el 27\% confirma utilizarlo diariamente o semanalmente para este fin. Por último, dentro de las escuelas el uso de los recursos tecnológicos con el objetivo de que los estudiantes realicen ejercicios del currículum (25\%) o para desarrollar los deberes escolares (20\%) es ligeramente menos común al registrado en usar el correo electrónico o chatear con los compañeros (26\%) (tabla 3).

Tabla 3. Frecuencia de utilización de los recursos tecnológicos dentro de la escuela, en porcentaje

Chat para hablar con los compañeros

Email para compartir trabajos con estudiantes y/o profesores

Internet para hacer los trabajos escolares

Internet para descargar información

Plataforma web del colegio para subir trabajos escolares

Simulaciones educativas

Ordenador para hacer ejercicios del currículo

Ordenador para hacer los deberes

Ordenador para trabajar en grupo

\begin{tabular}{cccc} 
CASI nUNCA & MENSUALMENTE & SEMANALMENTE & A DiARIO \\
\hline 66,21 & 7,94 & 8,29 & 17,56 \\
53,76 & 19,56 & 16,30 & 9,13 \\
27,38 & 27,13 & 28,64 & 15,43 \\
60,40 & 16,13 & 13,33 & 9,15 \\
71,75 & 12,68 & 8,54 & 6,23 \\
74,71 & 11,64 & 7,12 & 4,76 \\
51,03 & 22,88 & 15,46 & 9,55 \\
61,89 & 16,38 & 12,98 & 7,44 \\
44,36 & 28,26 & 17,64 & 8,93
\end{tabular}

Fuente: Elaboración propia 


\subsection{Incidencia de la utilización de los recursos tecnológicos en el aprendizaje}

Para dar respuesta al segundo de los objetivos planteados en esta investigación, determinar la relación entre la utilización de los recursos tecnológicos dentro de la escuela y el rendimiento académico de los estudiantes en matemáticas y lectura, se realiza un análisis multinivel con dos niveles de análisis. Este método de análisis permite conocer el impacto que la utilización de recursos tecnológicos tiene sobre el aprendizaje de los estudiantes descontando el efecto que generan las características previas de los estudiantes y del contexto donde se encuentra la escuela. Los resultados se encuentran en las tablas 4 y 5 .

De las cinco variables de ajuste utilizadas que se presentan en el modelo ajustado, cuatro realizan un aporte significativo confirmando su función de control en el modelo, excepto la asistencia del estudiante a preescolar que parece no hacer una aportación significativa al rendimiento en matemáticas de los estudiantes de educación secundaria. Es interesante confirmar la incidencia del nivel cultural y económico de la familia y del barrio donde se encuentre la escuela en los niveles de logro que los estudiantes alcanzan en ambas materias. Así como relevante resulta la brecha social con los inmigrantes, quiénes llegan a obtener 15 puntos menos en matemáticas y 12,7 puntos menos en lectura.

Los resultados de los modelos con las variables individuales (modelos III) indican la aportación que las variables explicativas generan sobre el rendimiento académico. De acuerdo con los resultados obtenidos, de las nueve variables explicativas utilizadas, cuatro parecen no hacer una aportación significativa al rendimiento de los estudiantes en matemáticas. Se trata de la utilización del correo electrónico para compartir trabajos con estudiantes y/o profesores, la utilización de Internet para realizar trabajos escolares, la utilización de la plataforma web de la escuela o la utilización del ordenador para realizar los deberes escolares.

Tabla 4. Resultados del proceso de modelamiento multinivel de dos niveles de análisis para Rendimiento en Matemáticas

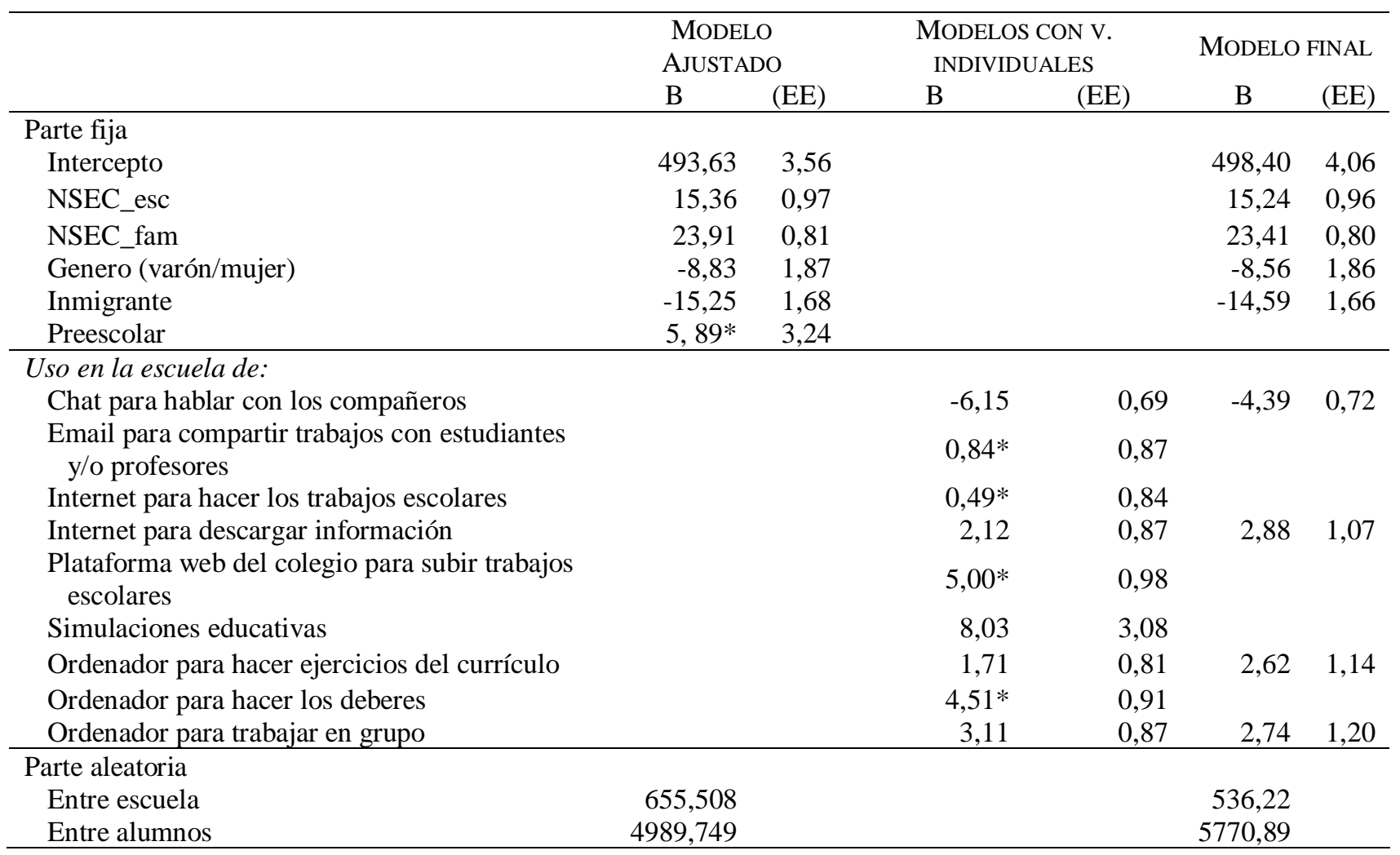

Nota: * No significativo a un $\alpha=0,05$.

Fuente: Elaboración propia

De las cinco variables que sí hacen una aportación significativa al modelo de rendimiento en matemáticas, destaca especialmente la mejora que genera en el rendimiento de los estudiantes 
utilizar simulaciones educativas en la docencia. Cuanto más se apoye el docente en este tipo de simulaciones complementando sus lecciones el rendimiento de los estudiantes aumenta 8 puntos. Por su parte, el rendimiento en matemáticas aumentará hasta 3 puntos por cuanto más frecuente sea la integración del ordenador como recurso tecnológico para la realización de trabajos en grupo, y casi 2 puntos si se utiliza para que los estudiantes realicen los ejercicios escolares o se consulte Internet para hacer búsquedas de información relativas con el temario abordado. Recordemos que el rendimiento en matemáticas tiene media 500, y desviación típica 100.

El modelo final (modelo IV) se construye de nuevo sobre el modelo ajustado, pero incorporando únicamente aquellas variables que han realizado una aportación significativa en los modelos III. De las cinco variables que hicieron una aportación significativa, cuatro forman parte del modelo final. Destaca la aportación positiva que realizan la utilización de Internet para consultar información y descargarla para apoyar los trabajos y/o las lecciones, la utilización del ordenador para hacer los ejercicios y para trabajar en grupo. La utilización del chat como recurso para que los estudiantes hablen con sus compañeros impacta de manera negativa sobre el rendimiento en matemáticas, 4 puntos menos cuanto más aumente la frecuencia de su uso

Tabla 5. Resultados del proceso de modelamiento multinivel de dos niveles de análisis para Rendimiento en Lectura

\begin{tabular}{|c|c|c|c|c|c|c|}
\hline & \multicolumn{2}{|c|}{$\begin{array}{l}\text { MODELO } \\
\text { AJUSTADO }\end{array}$} & \multicolumn{2}{|c|}{$\begin{array}{l}\text { MODELOS CON V. } \\
\text { INDIVIDUALES }\end{array}$} & \multicolumn{2}{|c|}{ MODELO FINAL } \\
\hline & $\mathrm{B}$ & $(\mathrm{EE})$ & $\mathrm{B}$ & $(\mathrm{EE})$ & $\mathrm{B}$ & $(\mathrm{EE})$ \\
\hline \multicolumn{7}{|l|}{ Parte fija } \\
\hline Intercepto & 555,70 & 3,68 & & & & \\
\hline NSEC_esc & 10,36 & 1,09 & & & & \\
\hline NSEC_fam & 23,09 & 0,83 & & & & \\
\hline Genero (varón/mujer) & $7,83 *$ & 3,94 & & & & \\
\hline Inmigrante & $-12,71$ & 1,73 & & & & \\
\hline Preescolar & $8,89 *$ & 4,93 & & & & \\
\hline \multicolumn{7}{|l|}{ Uso en la escuela de: } \\
\hline Chat para hablar con los compañeros & & & 2,10 & 1,01 & & \\
\hline $\begin{array}{l}\text { Email para compartir trabajos con estudiantes } \\
\text { y/o profesores }\end{array}$ & & & $-2,61 *$ & 1,98 & & \\
\hline Internet para hacer los trabajos escolares & & & $-0,89 *$ & 0,86 & & \\
\hline Internet para descargar información & & & $3,52 *$ & 1,89 & & \\
\hline $\begin{array}{l}\text { Plataforma web del colegio para subir trabajos } \\
\text { escolares }\end{array}$ & & & $1,38 *$ & 1,01 & & \\
\hline Simulaciones educativas & & & 15,69 & 1,10 & 7,01 & 1,31 \\
\hline Ordenador para hacer ejercicios del currículo & & & 6,11 & 2,89 & 3,23 & 1,09 \\
\hline Ordenador para hacer los deberes & & & $9,01 *$ & 0,94 & & \\
\hline Ordenador para trabajar en grupo & & & 3,88 & 0,92 & 2,02 & 0,89 \\
\hline \multicolumn{7}{|l|}{ Parte aleatoria } \\
\hline Entre escuela & 612,49 & & & & 336,22 & \\
\hline Entre alumnos & 5888,58 & & & & 5770,89 & \\
\hline
\end{tabular}

Nota: * No significativo a un $\alpha=0,05$.

Fuente: Elaboración propia

El modelo ajustado para rendimiento en lectura indica que tres de las cinco variables de ajuste hacen una aportación significativa al modelo. En este caso, el género del estudiante no genera un impacto sobre el rendimiento en lectura, tampoco la asistencia a preescolar. Como ocurría en el caso de rendimiento en matemáticas, destacan especialmente las aportaciones que el nivel socioeconómico y cultural de la familia del estudiante y del barrio donde se ubica la escuela generan sobre el rendimiento.

Los resultados de los modelos con las variables de ajuste apuntan a que, de las nueve variables que utiliza esta investigación, cinco parecen no hacer una aportación significativa al rendimiento en lectura. Se trata de las variables: utilización del correo electrónico para compartir trabajos con estudiantes y/o profesores, de Internet para hacer 
los trabajos escolares y para descargar información, de la plataforma web del colegio para subir trabajos escolares, y del ordenador para hacer los deberes.

Por otro lado, los juegos educativos interactivos hacen una aportación significativa al rendimiento de los estudiantes. La utilización del chat para mantener diálogos con los compañeros mejora el rendimiento en lectura de los estudiantes (2 puntos), también mejora el rendimiento cuando más aumente la frecuencia por utilizar el ordenador para hacer los ejercicios del tema de estudio (6 puntos por cada punto que aumente la frecuencia) y el uso del ordenador para trabajar en grupo $(3,9$ puntos de mejora).

En el modelo final tan solo tres de las variables han resultado hacer una aportación significativa al introducirse de manera conjunta sobre el modelo ajustado. La utilización de los recursos tecnológicos que más impacta sobre el rendimiento en lectura es la utilización de juegos educativos interactivos para la enseñanza, a continuación, la utilización de los ordenadores para realizar las los ejercicios de clase y su uso para trabajar en grupo.

\section{DISCUSIÓN Y CONCLUSIONES}

La presente investigación ha buscado dar respuesta a dos objetivos: describir la utilización que los estudiantes de educación secundaria dan a los recursos tecnológicos dentro y fuera de la escuela, y determinar cuál es la relación entre la utilización de los recursos dentro de la escuela y el rendimiento académico de los estudiantes en matemáticas y lectura.

La panorámica general aportada en la investigación sobre la utilización de los recursos tecnológicos por parte de los estudiantes dentro y fuera de las escuelas permite identificar que efectivamente a las innovaciones tecnológicas les queda un largo camino para estar presentes en la implementación del currículo. También ha demostrado la existencia de dos realidades, dentro y fuera de la escuela los recursos tecnológicos se utilizan de manera radicalmente diferente por los estudiantes. Mientras que durante el horario escolar destaca que menos de un $10 \%$ de los estudiantes utiliza los recursos tecnológicos a diario (a excepción del chat para hablar con sus compañeros), al encontrarse fuera de la escuela los estudiantes utilizan con mucha frecuencia la conexión a Internet para buscar información extra para las tareas escolares, descargar materiales y para la comunicación sincrónica y asincrónica a través de correos electrónicos o redes sociales entre los compañeros.

Estos resultados indican que los recursos tecnológicos están haciéndose cada vez más presentes en las aulas, aunque también que su utilización en el día a día de las aulas y la docencia está aún en desarrollo. Así mismo, pone en especial relevancia el papel de los estudiantes, quiénes parecen que serán los verdaderos impulsores de la implementación total de los recursos tecnológicos en las escuelas. Se trata de una mejora de abajoarriba, parece que será la demanda de los recursos tecnológicos por parte de los estudiantes, asociado a su nivel de destreza y dominio, quién será el verdadero impulsor del cambio en las escuelas.

Estos resultados son coherentes con los aportados por Area, Cepeda y Feliciano (2018) quienes señalan que la inmensa mayoría del alumnado de educación primaria y secundaria son una generación familiarizada con la tecnología digital, motivada y que demanda expresamente el uso de los recursos tecnológicos en la escuela, aunque también son críticos con el nivel de alfabetización digital alcanzado por sus profesores. Efectivamente, nuestros resultados indican que hay una escasa utilización, por ejemplo, de la plataforma web de la escuela, quizá porque ni los docentes ni los estudiantes la sientan del todo como suya, de manera que esté actualizada y sea realmente un recurso para la mejora.

Estudios como el realizado por Gil y Padilla (2016) demuestran lo relevante que es la cultura educativa y docente para el desarrollo de la competencia digital de los estudiantes. De manera que plataformas web no integradas, desactualizadas y con información de utilidad para las familias, se traducen en tareas administrativas que se suman al resto de obligaciones de los docentes, en vez de resultar un apoyo para la enseñanza de calidad. Los datos encontrados en la presente investigación demuestran la aportación significativa de los recursos tecnológicos dentro de las escuelas como apoyo al proceso de enseñanza-aprendizaje, concretamente se demuestra que los recursos que más impactan sobre aprendizaje son: la utilización del chat, el acceso a Internet para descargar información, la utilización de juegos/simulaciones educativas, la utilización del ordenador para hacer los ejercicios del currículo y trabajar en grupo. La tabla 6 recoge las variables explicativas que han realizado una aportación significativa en los análisis realizados. 
Tabla 6. Resumen de los Modelos Multinivel para Rendimiento en Matemáticas y Rendimiento en Lectura

\begin{tabular}{lcc}
\hline & RTO. MATEMÁTICAS & RTO. LECTURA \\
\hline Chat para hablar con los compañeros & $\mathrm{XX}$ & $\mathrm{X}$ \\
Email para compartir trabajos con estudiantes y/o profesores & \\
Internet para hacer los trabajos escolares & $\mathrm{XX}$ & \\
Internet para descargar información & $\mathrm{X}$ & $\mathrm{XX}$ \\
Plataforma web del colegio para subir trabajos escolares & $\mathrm{XX}$ & $\mathrm{XX}$ \\
Simulaciones educativas & & $\mathrm{XX}$ \\
Ordenador para hacer ejercicios del currículo & \\
Ordenador para hacer los deberes & \\
Ordenador para trabajar en grupo & & $\mathrm{XX}$ \\
\hline
\end{tabular}

Nota: X, la variable hace una aportación significativa en los Modelos III al incluirlas de manera individual en el modelo. XX, la variable hace una aportación significativa en el Modelo Final.

Fuente: Elaboración propia

Los resultados encontrados muestran que no hay un único modelo de innovación tecnológica en las escuelas. Los valores y las metas de cada escuela son los que marcan la idoneidad de los cambios y la utilización diferencial de los recursos tecnológicos. Para poder sacar su máximo beneficio en las escuelas, y lograr que ayuden al proceso formativo y mejoren la calidad de la educación, es fundamental que se refuerce la capacidad innovadora de todo el proceso educativo, su renovación y su análisis de aquello que le falta por ofrecer para atender de mejor manera a sus estudiantes. De esta forma es necesario, primero, identificar el uso que se le está dando a los recursos tecnológicos para, a continuación, estudiar el efecto que generan sobre los aprendizajes. Con el estudio realizado se da respuesta a ambos interrogantes, sin embargo, se abren muchos más. De un lado, si los recursos, como se han visto, están presentes en las aulas es fundamental preguntarse por la calidad de la formación permanente del profesorado y evitar la clásica formación autodidacta, así como la formación promovida desde las editoriales que se asocia al negocio de la educación. Los docentes necesitan una formación ajustada a sus necesidades, intereses y coherente con garantizar una enseñanza de calidad para todas y todos los estudiantes atendiendo a su diversidad. Si tal y como señalan Prendes et al. (2018) los futuros maestros han integrado los recursos tecnológicos en la comunicación, resulta fundamental que integren sus competencias digitales en su trabajo académico.

También conocer el efecto que la utilización de los recursos tecnológicos genera sobre el rendimiento de los estudiantes en educación primaria para, en tal caso, poder contribuir cuanto antes en el desarrollo académico de los estudiantes. $\mathrm{Y}$ ahondar en las causas del bajo uso de las plataformas educativas. La variedad de casuísticas, casi tantas como escuelas, invitan a un abordaje cualitativo que identifique los indicadores de calidad de las plataformas educativas para los estudiantes, sus familias y los docentes, y analizarlas en función de los patrones de calidad aportados por investigaciones previas como Ardila (2011). Por supuesto, resulta especialmente relevante que se desarrollen trabajos que aborden la relación entre la utilización de los recursos tecnológicos fuera de las escuelas, la investigación educativa actual y las políticas educativas de manera que se ahonde en el diseño de acciones educativas próximas a los intereses de los estudiantes, que contribuyan a la enseñanza del currículo y que garanticen la inclusión de todos los estudiantes atendiendo a la diversidad de sus competencias.

Esta investigación ha demostrado que una amplia utilización de los recursos tecnológicos asociados al currículo escolar incide sobre el desarrollo cognitivo de los estudiantes. Es decir, son un factor de enseñanza eficaz. Si las escuelas no disponen de los recursos didácticos necesarios para que la docencia se lleve a cabo con calidad, o si el profesorado no está instruido en su utilización, el impacto de los recursos se merma. Si estamos comprometidos con ofrecer una enseñanza de calidad a los estudiantes, es fundamental una acción conjunta a nivel estatal que potencie la ajustada utilización de los recursos tecnológicos en todas las escuelas.

\section{REFERENCIAS}

Agodini, R., Dynarski, M., Honey, M., y Levin. D. (2003). The effectiveness of educational technology: Issues and recommendations for the national study. Washington, DC: Mathematica Policy Research Inc,

Aldama, C., y Pozo, J. C. (2016). How are ICT used in the classroom? A study of teachers' beliefs and uses. 
Electronic Journal of Research in Educational $\begin{array}{lll}\text { Psychology, } & \text { 14(2), }\end{array}$ https://doi.org/10.14204/ejrep.39.15062

Area, M. (2007). Algunos principios para el desarrollo de buenas prácticas pedagógicas con las TIC en el aula. Revista Comunicación y Pedagogía, 222, 42-47.

Area, M., Cepeda, O. y Feliciano, L. (2018). El uso escolar de las TIC desde la visión del alumnado de Educación Primaria, ESO y Bachillerato. Educatio XXI, 36(2), 229-276. https://doi.org/ 10.6018/j/333071

Ardila, M. (2011). Indicadores de calidad de las plataformas educativas digitales. Educación y Educadores, 14(1), 189-206.

Balanskat, A., Blamire, R., y Kefala, S. (2006). The ICT impact report: a review of studies of ICT impact on schools in Europe. Bruselas: European Communities.

Baztan, M. (2014). Los materiales didácticos digitales en la enseñanza no universitaria española. Valencia: Ediciones Universitat Politécnica de València.

Bonilla, P. J. S., Nemiña, R. E., y Suelves, D. M. (2017). Análisis y evaluación de portales institucionales en España. Los casos de Canarias, Galicia y Valencia. RELATEC: Revista Latinoamericana de Tecnología Educativa, 16(2), 29-48.

Cervini, R. (2009). Class, school, municipal, and state effects on mathematics achievement in Argentina: A multilevel analysis. School Effectiveness and School Improvement, 20(3), 319-340. https://doi.org/10.1080/09243450802664404

Creemers, B.P.M. (1994). The effective classroom. Londres: Cassell.

Fernández-Miravete, A. D. (2018). La competencia digital del alumnado de Educación Secundaria en el marco de un proyecto educativo TIC (1:1). EDUTEC. Revista Electrónica de Tecnología Educativa, 63, 60-72. https://doi.org/10.21556/edutec.2018.63.1027

Gairín Sallán, J., y Mercader, C. (2018). Usos y abusos de las TIC en los adolescentes. Revista de Investigación Educativa, $\quad 36(1), \quad 125-140$. http://dx.doi.org/10.6018/rie.36.1.284001

Garrison, M., y Bromley, H. (2004). Social contexts, defensive pedagogies, and the (mis)uses of educational technology. Educational Policy, 18(4), 589-613. https://doi.org/10.1177/0895904804266643

Genlott, A. A., y Grönlund, Å. (2016). Closing the gapsImproving literacy and mathematics by ict-enhanced collaboration. Computers \& Education, 99, 68-80. https://doi.org/10.1016/j.compedu.2016.04.004

Gil, J. M., y Padilla, P. (2016). La competencia digital en la educación secundaria: ¿dónde están los centros? Aportaciones de un estudio de caso. New Approaches in Educational Research, 5(1), 60-66.

Hammond, M. (2014). Introducing ICT in schools in England: Rationale and consequences. British Journal of Educational Technology, 45(2), 191-201. https://doi.org/10.1111/bjet.12033

Huertas Montes, A., y Pantoja Vallejo, A. (2016). Efectos de un programa educativo basado en el uso de las TIC sobre el rendimiento académico y la motivación del alumnado en la asignatura de tecnología de educación secundaria. Educación XX1, 19(2), 229-250.

Lizasoain, L., y Joaristi, L. (2012). Las nuevas tecnologías y la investigación educativa. El análisis de datos de variables categoriales. Revista Española de Pedagogía, 15(251), 111-130.
Marqués, P. (2013). Impacto de las TIC en la educación: Funciones y limitaciones. Revista de Investigación 3Ciencias, 2(1), 1-15.

Murillo, F. J. (2007). Investigación iberoamericana sobre eficacia escolar. Bogotá: Convenio Andrés Bello.

Murillo, F. J., Martínez-Garrido, C., y Hernández Castilla, R. (2011). Decálogo para una enseñanza eficaz. REICE. Revista Iberoamericana sobre Calidad, Eficacia y Cambio en Educación, 9(1), 6-27.

Martínez-Garrido, C., y Murillo, F. J. (2016). investigación iberoamericana sobre enseñanza eficaz. Revista Mexicana de Investigación Educativa, 21(69), 471499.

Paiva, J. (2005). ICT uses in educational context: Some data and reflections on portuguese reality. Interactive Educational Multimedia, 11, 27-37.

Pantoja, A., y Villanueva, C. (2015). Mejoras del conocimiento de la cultura propia y del otro tras la aplicación de un programa basado en las TIC. Revista de Investigación Educativa, 33(1), 133-148. https://doi.org/10.6018/rie.33.1.191591

Pérez, A. I., y Sola, M. (2006). La emergencia de buenas prácticas. Sevilla: Junta de Andalucía.

Prendes, M. P., Solano, I. M., Serrano, J. L., González, V., y Mar Román, M. (2018). Entornos personales de aprendizaje para la comprensión y desarrollo de la competencia digital: Análisis de los estudiantes universitarios en España. Educatio Siglo XXI, 36(2), 115-134. https://doi.org/10.6018/j/333081

Reedy, G. (2008). PowerPoint, interactive whiteboards, and the visual culture of technology in schools. Technology Pedagogy and Education, 17(2), 143-162. https://doi.org/10.1080/14759390802098623

Reynolds, D., Creemers, B., Nesselrodt, P. S., Shaffer, E. C., Stringfield, S., y Teddlie, C. (Eds.). (2014). Advances in school effectiveness research and practice. Londres: Elsevier. https://doi.org/10.1016/c2009-0-14863-8

Sánchez-Antolín, P., y Blanco-García, M. M. (2016). La política educativa TIC de la Comunidad de Madrid (España): La perspectiva del profesorado. Revista Latinoamericana de Tecnología Educativa, 15(1), 4558.

Scheerens, J. (2013). Un meta-análisis de estudios de eficacia escolar. Revista de Educación, 361, 1-25.

Selwyl, N. Potter, J. y Cranmer, S. (2000). Primary pupils' use of information and communication technologies at school and home. British Journal of Educational Technology, 40(5), 919-932. https://doi.org/10.1111/j.1467-8535.2008.00876.x

Sosa, M.J., Peligros, S., y Dionisio Díaz, M. (2010). Buenas prácticas organizativas para la integración de las TIC en el sistema educativo extremeño. Teoría de la Educación en la Sociedad de la Información, 11(1), 148-179.

Valverde-Berrocoso, J. y Sosa-Díaz, M. J. (2014). Centros educativos e-competentes en el modelo 1:1. El papel del equipo directivo, la coordinación TIC y el clima organizativo. Profesorado. Revista de Currículum y Formación del Profesorado, 18(3), 41-62. 